\title{
Cáncer de mama - 1982-1993
}

\author{
Mario González* Gustavo Marquez**
}

\section{RESUMEN}

OBJETIVO: Evaluar las características personales, parámetros, pronósticos, tratamientos y sobrevida de los pacientes con cáncer de mama en el período de 1982 a 1993 en la C.S.P.C. de Santafé de Bogotá (I.S.S.).

MATERIAL Y METODOS: Se organizan y resumen los datos de características personales y clínicas, tratamiento y sobrevida con base en el TNM incorporando las pacientes a la última clasificación por estados.

Se analizaron frecuencias con el programa EPIINFO, la sobrevida se estimó con el método de Kaplan-Meier y la comparación de sobrevida mediante el de Mantzel-Haenszel, ambos métodos con el programa TRUE-EPISTAT eligiendo un valor de p menor de 0.05 como significativo.

RESULTADOS: Se encontraron 488 pacientes con promedio de edad 48,16 años, el $46 \%$ menopáusicas, tamaño tumoral mayor de 5 cms. en el $44 \%$ de los casos siendo el carcinoma ductal infiltrante el tipo histológico más frecuente $80.7 \%$. Estados IIIA el $24 \%$, IIIB el $24.1 \%$ y IV el $7.4 \%$. La mastectomía radical modificada fue el tipo de cirugía más realizado $(90 \%)$. Las variables con significancia estadística en la sobrevida y el tiempo libre de enfermedad fueron: estado, niveles y total de ganglios axilares comprometidos, tipo de recaída y tamaño del tumor.

CONCLUSIONES: Este estudio permitió establecer que los factores pronósticos con significancia estadística en el análisis de sobrevida y tiempo libre de enfermedad son los mismos encontrados en otros estudios. Estos tienen valor en la decisión terapéutica y en la investigación del carcinoma mamario.

\section{Introducción}

El cáncer de mama representa el $19 \%$ de todas las causas de mortalidad por cáncer en las mujeres (9). Algunos reportes lo ubican como el cáncer más frecuente en la mujer, siendo el $27 \%$ de todos los cánceres (9).

En Colombia, se nota un incremento estadístico a través de los informes del Plan Nacional de Cáncer, siendo solamente superado por el carcinoma de cuello uterino y el de piel $(5,15)$.

El cáncer de mama se ha constituido en un gran reto dentro del conocimiento médico, para ser debido a toda una constelación de factores epidemiológicos, incluyendo predisposición genética, medio hormonal adverso, incompetencia inmunológica, exposición a carcinógenos y diversas situaciones adversas personales y demográficas. El desconocimiento de los factores implicados conlleva a que solamente el $20 \%$ de las mujeres con esta patología tenga uno de los factores de riesgo identificados actualmente.

Una vez establecido el cáncer de mama, existen diversos parámetros pronósticos que parecen basarse en una interacción dinámica entre la extensión anatómica del cáncer (12). El momento en que se llevó a cabo el diagnóstico y el potencial de crecimiento o la virulencia

\footnotetext{
* Médico ginecoobstetra, Clínica San Pedro Claver

** Médico, Clínica San Pedro Claver
}

de los tumores por un lado, y el grado de inmunocompetencia del huésped y el tratamiento más idóneo por el otro.

El tratamiento del cáncer de mama implica un manejo multidisciplinario en el que la cirugía representa la modalidad de primer orden en lo que respecta a la certeza diagnóstica, la remoción del volumen tumoral y la extirpación de los tejidos tumorales con fines de evaluación pronóstica $(4,11)$.

\section{Objetivos}

Evaluar las características personales, parámetros, pronósticos, tratamientos y sobrevida de las pacientes con cáncer de mama en el período de 1982-1993 en la Clínica San Pedro Claver de Bogotá.

\section{Material y métodos}

Se realizó una revisión de la historia clínica de las pacientes con diagnóstico de cáncer de mama en el Servicio de Oncología de la Clínica San Pedro Claver (ISS), Santafé de Bogotá, de 1982 a 1993.

Se organizan y resumen los datos de características personales, clínicas, tratamiento y sobrevida. Con base en los datos del TNM, se incorporaron las pacientes a la última clasificación por estados (1988), Comisión Conjunta del Cáncer de los Estados Unidos (ACJJ) y la Unidad Internacional contra el Cáncer (UICC). 
Se realizó análisis de frecuencias con el programa EPIINFO, la sobrevida se estimó con el método de KAPLAN-MEIER y la Comparación de sobrevidas mediante el método de MANTEL-HAENSZEL, realizados con el programa TRUE-EPISTAT eligiendo un valor de $\mathrm{P}<0,05$ para determinar la significancia estadística.

\section{Resultados}

\section{Edad}

Su distribución dentro del total de 488 pacientes mostró una media de 48,16 años, el 3,7\% fue menor de 30 años y el $16 \%$ mayor de 60 años.

\section{Menarquia}

El $27 \%$ de las pacientes tuvieron menarquia antes de los 12 años. En general, la media fue de 13,3 años.

\section{Menopausia}

El $46 \%$ de las pacientes con carcinoma mamario correspondió a pacientes en período de menopausia. La edad media de menopausia fue de 47 años.

\section{Síntomas}

El síntoma predominante fue el dolor el cual estuvo presente en el $94,3 \%$ de las pacientes, otros síntomas fueron: picadas, fiebre, prurito, dolor óseo, etc.

El signo de mayor hallazgo fue la presencia de masa en 402 pacientes $(83,4 \%)$ los demás signos (secreción serosa, sanguinolenta, calor, rubor) en general de inflamación fueron muy variados y se manifestaron en escasa proporción frecuentemente acompañando la masa.

\section{Anticoncepción}

Solamente 69 pacientes reportaron antecedentes de uso de anticonceptivos.

\section{Edad de primer parto}

Hubo 138 pacientes (26\%) nulíparas. El 3,7\% tuvieron su primer parto antes de los 15 años y el 2,6\% después de los 35 años de edad, para un total de 350 pacientes.

\section{Tamaño tumoral}

En el $40,4 \%$ de los casos estuvo entre 2 y $5 \mathrm{cms}$., en el $15,5 \%$ menor de $2 \mathrm{~cm}$. y el resto o sea el $44,1 \%$ mayor de 5 cm., siendo el mayor tamaño tumoral de $22 \mathrm{cms}$ (Tabla 1).

\section{Localización tumoral}

La localización tumoral más frecuente del tumor es el cuadrante supero-externo en el $42,33 \%$ seguida de la del cuadrante supero interno con el $18,2 \%$, en el $15,7 \%$ el compromiso fue de los dos cuadrantes (tabla 2).

\section{Sitio de Metástasis}

Se presentó metástasis a distancia en 150 casos siendo en su mayoría a nivel óseo ( $30 \%$ de ellas) seguido por compromiso pulmonar $(21,3 \%)$ y de hígado $(6,7 \%)$.
Tabla 1

TAMAÑO TUMORAL

\begin{tabular}{|c|c|c|}
\hline TAMANO & N & PORCENTAJE \\
\hline$<2 \mathrm{~cm}$. & 70 & 15.5 \\
\hline $3.5 \mathrm{~cm}$. & 182 & 40.4 \\
\hline$>5 \mathrm{~cm}$. & 199 & 44.1 \\
\hline TOTAL & 451 & $100 \%$ \\
\hline
\end{tabular}

Tabla 2

LOCALIZACION DEL TUMOR

\begin{tabular}{|c|c|c|}
\hline LOCALIZACION & $\mathbf{N}$ & PORCENTAJE \\
\hline Cuadrante supero - externo (CSE) & 205 & 42.3 \\
\hline Cuadrante supero - interno (CSI) & 88 & 18.1 \\
\hline Cuadrante infero - interno (CII) & 22 & 4.5 \\
\hline Cuadrante infero - externo ( $\mathrm{ClE}$ ) & 39 & 8.0 \\
\hline \begin{tabular}{|l|l|} 
Retroareolares \\
\end{tabular} & 8 & 1.6 \\
\hline Pezón & 1 & 0.2 \\
\hline Totalidad & 11 & 2.2 \\
\hline CSE - CSI & 50 & 10.3 \\
\hline CSI-CII & 4 & 0.9 \\
\hline $\mathrm{CII}-\mathrm{CIE}$ & 12 & 2.5 \\
\hline CSE - CIE & 10 & 2.0 \\
\hline Otras & 35 & 7.2 \\
\hline TOTAL & 484 & $100 \%$ \\
\hline
\end{tabular}

\section{Compromiso ganglionar}

Nivel 1. De las disecciones realizadas en 329 pacientes, 175 tuvieron compromiso tumoral en este nivel.

Este fue de 1 ganglio en 26 pacientes, y el máximo compromiso fue de 21 ganglios, en una de ellas. Se encontraron de 1 a 3 ganglios positivos en 71 pacientes, y el resto más de tres ganglios.

Nivel II. 14 de las 225 pacientes, con disección en este nivel presentaron compromiso de un sólo ganglio. En el grupo de 1 a 3 ganglios positivos para tumor hubo 35 pacientes.

Nivel III. De las 205 pacientes a quienes se les realizó disección ganglionar en el nivel III, 68 presentaron compromiso tumoral, 43 pacientes tuvieron positivos 1 a 3 ganglios.

\section{Diagnóstico de patología}

El carcinoma ductal infiltrante fue el diagnóstico más frecuente, se presentó en el 80,7\% de las pacientes de este estudio. Le siguió el carcinoma lobulillar $(7,7 \%)$, el medular $(3,0 \%)$ y el papilar $(3,2 \%)$ entre otros tipos histológicos estuvieron el Histiocítico, Paget, Células claras, Philodes, Fibrosarcoma, Angiosarcoma, etc. (Tabla 3).

\section{Estados clínicos}

La mayoría de los pacientes se diagnosticaron en estados avanzados de la enfermedad (55\%), siendo los estados IIIA y IIIB los frecuentemente encontrados, cada uno con el $24,1 \%$ y el IV $7,4 \%$.

En los estados tempranos se presentó la distribución que aparece en la tabla 4. 
Tabla 3

TIPO HISTOPATOLOGICO

\begin{tabular}{|c|c|c|}
\hline TIPO HISTOPATOLOGICO & N & PORCENTAJE \\
\hline & & \\
\hline DUCTAL & 245 & 80.7 \\
\hline LOBULILLAR & 33 & 7.7 \\
\hline PAPILAR & 14 & 3.2 \\
\hline MEDULAR & 13 & 3.0 \\
\hline OTROS & 22 & 5.1 \\
\hline TOTAL & & $99.7 \%$ \\
\hline
\end{tabular}

Tabla 4

ESTADO CLINICO

\begin{tabular}{||c|c|c|}
\hline ESTADO & N & PORCENTAJE \\
\hline I & & \\
\hline IIA & 28 & 6.0 \\
\hline II B & 87 & 18.6 \\
\hline III $A$ & 19.8 \\
\hline III B & 93 & 24.1 \\
\hline IV & 113 & 24.1 \\
\hline & 113 & 7.4 \\
\hline TOTAL & 34 & \\
\hline
\end{tabular}

\section{Historia familiar}

En 54 pacientes correspondientes al $12,5 \%$ del total se encontraron antecedentes familiares de cáncer mamario.

\section{Sitio de recurrencia}

La recurrencia fue única en $88 \%$ de los casos. El principal sitio de recurrencia fue la pared torácica con el $33,9 \%$. En el $23,2 \%$ de los casos de cirugía conservadora se presentó en mama. En el 14,3\% del total hubo compromiso de ganglios supraclaviculares.

\section{Metástasis}

En el 70\% de los casos (341) pacientes hubo metástasis única.

\section{Receptores estrogénicos}

Se evaluaron al respecto 204 pacientes encontrándose niveles menores de 10 fmoles en el $55,4 \%$ de ellas, entre 10 y 50 fmoles, en el $38,6 \%$, entre 51 y 100 fmoles en el $4 \%$ y mayores el $2 \%$.

\section{Cirugía}

Se realizó en 396 pacientes, mastectomía radical modificada en el $90 \%$ de ellas, mastectomía simple en el 6,2\% y cuadrantectomía y resección axilar en el 3,8\% de pacientes. Esta última se realizó en 8 pacientes con estado I, en 6 con estado IIA y en 1 con estado IIB. (Tabla 5).

\section{Cobaltoterapia}

La recibieron 318 pacientes, una dosis menor de 5000 CGY el $76 \%$, de 5000 a 6000 CGY el $23.3 \%$ y sólo 2 pacientes $(0,6 \%)$ una dosis mayor. Tabla 6 .
Tabla 5

CIRUGIA

\begin{tabular}{|c|c|c|}
\hline TIPO DE CIRUGIA & $\bar{N}$ & PORCENTAJE \\
\hline Mastectomia radical modificada & 353 & 89.5 \\
\hline Mastectomia simple ( Total ) & 23 & 5.9 \\
\hline Cuadrantectomia & 15 & 3.8 \\
\hline Tumorectomia & 3 & 0.8 \\
\hline TOTAL & 395 & $100 \%$ \\
\hline
\end{tabular}

Tabla 6

DOSIS DE RADIOTERAPIA

\begin{tabular}{|c|c|c|}
\hline DOSIS (cGY) & N & PORCENTAJE \\
\hline & & \\
\hline$<5000$ & 242 & 76.1 \\
\hline $5000-6000$ & 74 & 23.3 \\
\hline$>6000$ & 2 & 0.6 \\
\hline TOTAL & 318 & $100 \%$ \\
\hline
\end{tabular}

\section{Receptores de progesterona}

Se evaluaron al respecto 202 pacientes, presentaron niveles menores de 10 fmoles el $70,8 \%$; entre 10 y 50 fmoles el $26,7 \%$ y tan sólo 5 pacientes $(2,5 \%)$ niveles mayores.

\section{Quimioterapia neoadyuvante}

Se administró a 184 pacientes, al 45,1\% CMF y al $45,3 \%$ FAC y al resto otras combinaciones.

\section{Quimioterapia adyuvante}

De los 262 pacientes en quienes se administró el mayor número de ellas 110 (45\%) recibieron una dosificación mayor a 6 ciclos de CMF y el resto CMF en menor número o bien FAC.

\section{Distribución de estados clínicos por edad}

La mayoría de pacientes se distribuyeron en el grupo de enfermedad localmente avanzada (estado III) $48 \%$.

Se encontró una asociación estadísticamente significativa $\mathrm{P}<0,01$, entre estados clínicos avanzados y mayor edad.

\section{Edad y tamaño tumoral}

Para los grupos de edad media de 40 a 60 años se encontró una asociación estadísticamente significativa $\mathrm{P}<0,05$ con los tamaños tumorales mayores de 5 centímetros, siendo su distribución sensiblemente igual para el grupo de 40 a 50 años y el de 51 a 60 años.

\section{Menarquia y tamaño tumoral}

El tamaño tumoral mayor mostró una asociación estadísticamente significativa $\mathrm{P}<0,04$ en los pacientes con menarquia después de los 13 años. 


\section{Menopausia y estado clínico}

El grupo de pacientes premenopáusicas se asoció en forma estadísticamente significativa con los estados clínicos avanzados al igual que con un mayor compromiso tumoral ganglionar $\mathrm{P}<0,01$.

El perfil ginecológico mostró una relación inversa entre la paridad y el número de ganglios positivos.

\section{Localización y estado clínico}

Se asoció la localización con el estado clínico mostrando mayor compromiso de los cuadrantes externos en todos los estados, con un valor de $\mathrm{P}<0,01$.

El tipo histológico mostró asociación importante con el número de ganglios positivos y el compromiso metastásico, siendo el carcinoma canalicular infiltrante el que mostró mayor compromiso ganglionar $\mathrm{P}<0,01 \mathrm{y}$ mayor grado de metástasis $\mathrm{P}<0,05$.

Se registró claramente una relación entre los estados clínicos localmente avanzados y la metástasis de presentación única, que correspondió al $70 \%$ de las pacientes en compromiso metastásico.

El presente estudio mostró una relación directa entre receptores al encontrarse bajos niveles estrogénicos y progestacionales simultáneamente.

\section{Sobrevida}

Las variables analizadas fueron sometidas a la prueba de Mantel-Haenszel para determinar su significancia estadística. Las pruebas con resultado significativo fueron estado, ganglios positivos y su compromiso tumoral de acuerdo con la distribución por niveles, tipos de recaída, tamaño tumoral y edad de menopausia. (Tabla 7 y figs. 2 a 10).

La sobrevida a cinco años por estado clínico fue menor por los estados IIIB $(50,8 \%)$ y IV $(25,08 \%)$ con un valor de $\mathrm{P}<0,001$.

Sólo el 33,3\% de los pacientes con recaída local múltiple estaban vivas a los cinco años, mientras que el $78,9 \%$ de aquellas con recaída local única lo estaba $\mathrm{P}<0,0001$.

Otras variables evaluadas que no fueron significativas en el análisis de sobrevida, se presentan en la tabla 8 .

En estas tablas, $\mathrm{N}$ corresponde al número total de pacientes por cada atributo; el porcentaje y el número de pacientes vivas a los 5 años y $\mathrm{P}$ el valor de significancia estadística de acuerdo con la comparación de sobrevidas.

En la tabla 9 le presentan factores de sobrevida por meses hasta 120 meses (10 años) de acuerdo con las diferentes variables y su respectiva sobrevida global, la cual se puede apreciar en la figura 1.

En ella se evidencia que la pendiente de la curva es mayor en los 3 primeros años después del diagnóstico, lográndose un nivel estable después de los 8 años.

\section{Tiempo libre de enfermedad}

En el análisis de tiempo libre de enfermedad las variables con significancia estadística fueron: Estado, total de ganglios positivos y su compromiso por niveles,
Tabla 7

PRONOSTICO SEGUN CARACTERISTICAS DE LAS PACIENTES SOBREVIDA

\begin{tabular}{|c|c|c|c|c|c|}
\hline \multirow{2}{*}{\multicolumn{2}{|c|}{ CАRAC:TICKISTILA }} & \multirow{3}{*}{$\frac{N}{28}$} & \multicolumn{2}{|c|}{ YIYAS A 5 ANOS } & \multirow{2}{*}{ Valor P } \\
\hline & & & $\%$ & $N$ & \\
\hline \multirow{6}{*}{ |(LST'AI) } & 1* & & 100,0 & 28 & \multirow[b]{6}{*}{$<0,0001$} \\
\hline & $\operatorname{IIA}$ & 87 & 87,3 & 76 & \\
\hline & III & 93 & $81, .5$ & 70 & \\
\hline & III & 113 & 80,3 & 91 & \\
\hline & 11113 & 113 & 50,8 & 57 & \\
\hline & IV & 34 & 25,8 & 9 & \\
\hline \multirow{4}{*}{ ESTADO } & 1* & 28 & 100 & $2 k$ & \multirow[b]{4}{*}{$<0,0001$} \\
\hline & II & 180 & 84,2 & 152 & \\
\hline & III & 226 & 65,4 & 148 & \\
\hline & IV & 34 & 25,8 & 9 & \\
\hline \multirow{3}{*}{$\begin{array}{l}\text { GANGLIOS POSITIVOS } \\
\text { NIVEL I }\end{array}$} & 0 & 154 & 92,6 & 143 & \multirow[b]{3}{*}{$<0,0001$} \\
\hline & $1-3$ & 77 & 82,7 & 64 & \\
\hline & $>3$ & 98 & 55,6 & 55 & \\
\hline \multirow{3}{*}{$\begin{array}{l}\text { GANGLIOS POSITIVOS } \\
\text { NIVEL } 2\end{array}$} & 0 & 128 & 87,7 & 112 & \multirow[b]{3}{*}{$<0,0001$} \\
\hline & $1-3$ & 35 & 73,9 & 26 & \\
\hline & $>3$ & 62 & 58,8 & 36 & \\
\hline \multirow{3}{*}{$\begin{array}{l}\text { GANGLIOS POSITIVOS } \\
\text { NIVEL } 3\end{array}$} & 0 & 137 & 86,4 & 118 & \multirow[b]{3}{*}{$<0,0001$} \\
\hline & $1-3$ & 43 & 64,5 & 28 & \\
\hline & $>3$ & 25 & 57,1 & 14 & \\
\hline \multirow{4}{*}{$\begin{array}{l}\text { TOTAL GANGLIOS } \\
\text { POSITIVOS }\end{array}$} & 0 & 153 & 91,6 & 140 & \multirow[b]{4}{*}{$<0,0001$} \\
\hline & $1-3$ & 64 & 80,1 & 51 & \\
\hline & $4-12$ & 77 & 69,2 & 53 & \\
\hline & 213 & 49 & 53,8 & 26 & \\
\hline \multirow{4}{*}{ TIPO DE RECAIDA } & Local única & 25 & 78,9 & 20 & \multirow{4}{*}{$<0,0001$} \\
\hline & Local múltiple & 3 & 33,3 & 1 & \\
\hline & $\begin{array}{l}\text { Metástasis } \\
\text { única }\end{array}$ & 80 & 31,6 & 25 & \\
\hline & $\begin{array}{l}\text { Metástasis } \\
\text { múlt. }\end{array}$ & 40 & 14,6 & 6 & \\
\hline \multirow{3}{*}{ TAMAÑO DEL TUMOR } & $\leq 2 \mathrm{cms}$ & 70 & 79,6 & 56 & \multirow[b]{3}{*}{0,002} \\
\hline & $3-5 \mathrm{cms}$ & 182 & 81,5 & 148 & \\
\hline & $>5 \mathrm{cms}$ & 199 & 66,3 & 132 & \\
\hline \multirow{3}{*}{ EDAD MENOPAUSIA } & $\leq 50$ años* & 13 & \multicolumn{2}{|c|}{ Insuficiente } & \\
\hline & $>50$ años & 192 & 78.9 & 152 & \\
\hline & No menopáus. & 265 & 69,8 & 185 & $0 ; 025$ \\
\hline
\end{tabular}

* No incluido en el análisis estadistico

tamaño del tumor, tipo de recaída, tipo histopatológico. (Tabla 10 Figs. 11 a 20).

El tiempo libre de enfermedad a cinco años por estado clínico mostró un $\mathrm{P}<0,001$, siendo para el estado IIIA de $59,9 \%$ y $38,0 \%$ para el IIIB.

De 34 pacientes en estado IV, ninguna estuvo libre de enfermedad al final de este período.

Según el tamaño tumoral de aquellas mayores de 5 cms. sólo el $49 \%$ estaban libres de enfermedad a los cinco años mientras lo estaban el 74,9\% de quienes habían presentado lesiones menores de $2 \mathrm{cms} \mathrm{P}<0,001$. 
Figura 1

SOBREVIDA GLOBAL

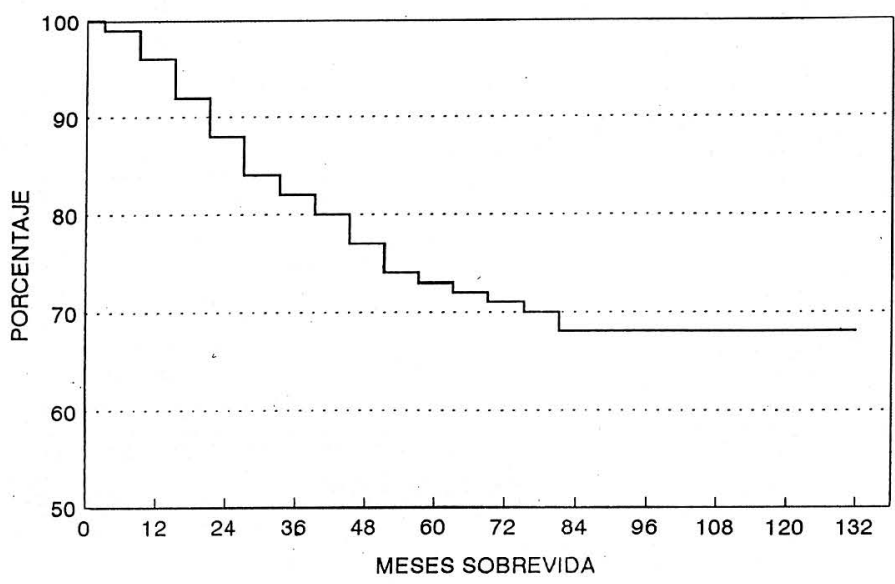

Figura 2

SOBREVIDA SEGUN ESTADO

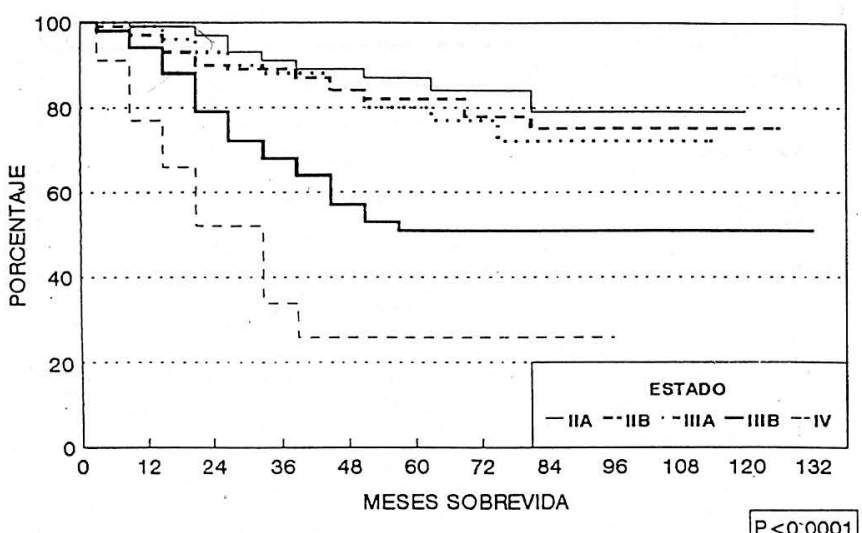

Figura 3

SOBREVIDA SEGUN ESTADO

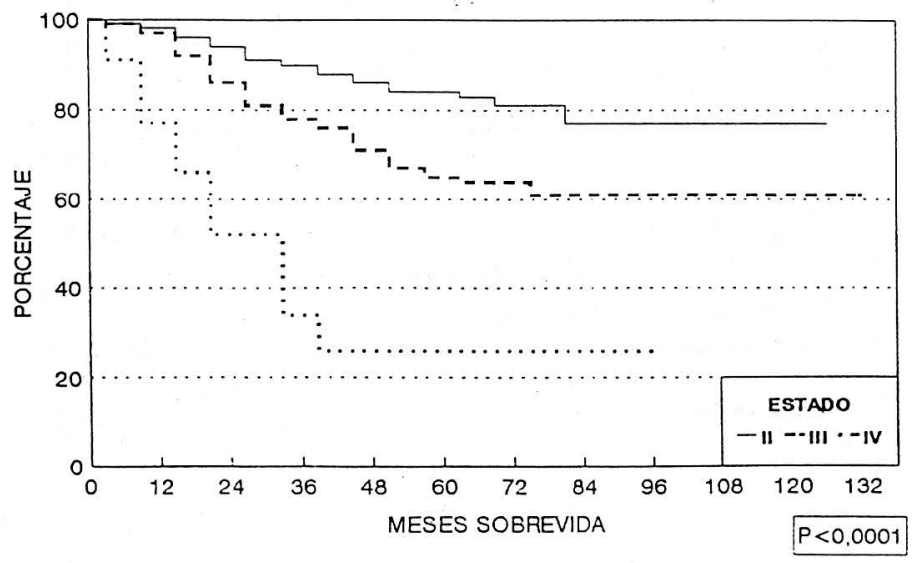

Figura 4

SOBREVIDA SEGUN NUMERO DE GANGLIOS POSITIVOS NIVEL 1

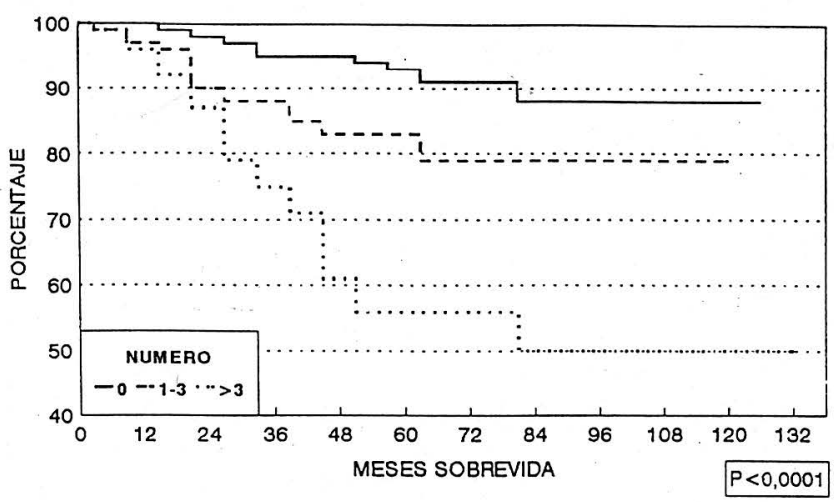

Figura 5

SOBREVIDA SEGUN NUMERO DE GANGLIOS POSITIVOS NIVEL 2

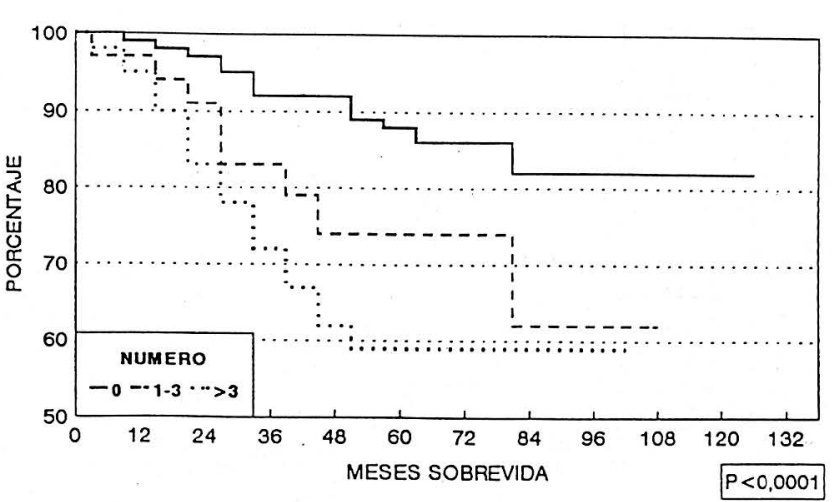

Figura 6

SOBREVIDA SEGUN NUMERO DE GANGLIOS POSITIVOS NIVEL 3

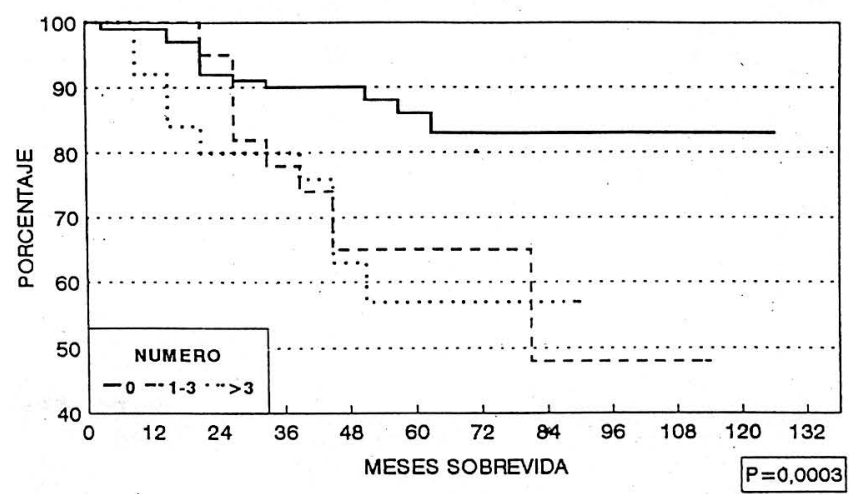


Figura 7

SOBREVIDA SEGUN TOTAL DE GANGLIOS POSITIVOS

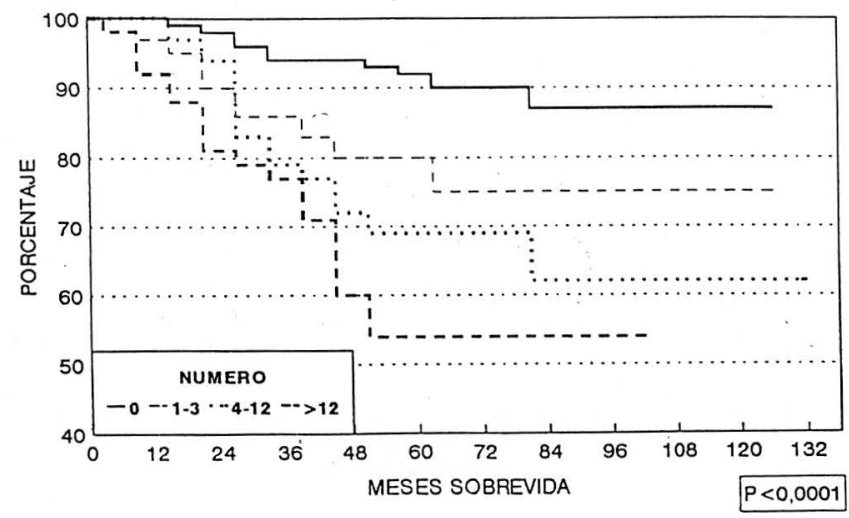

Figura 8

SOBREVIDA SEGUN TIPO DE RECAIDA

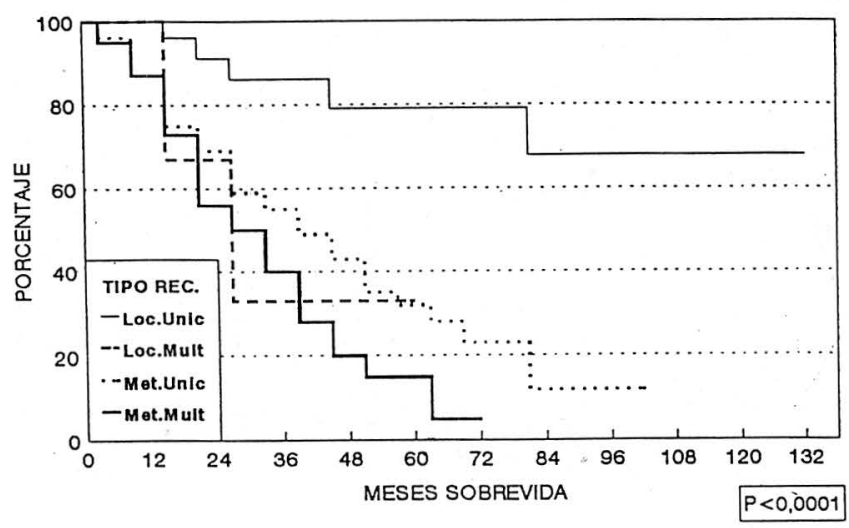

Figura 9

SOBREVIDA SEGUN TAMAÑO DEL TUMOR

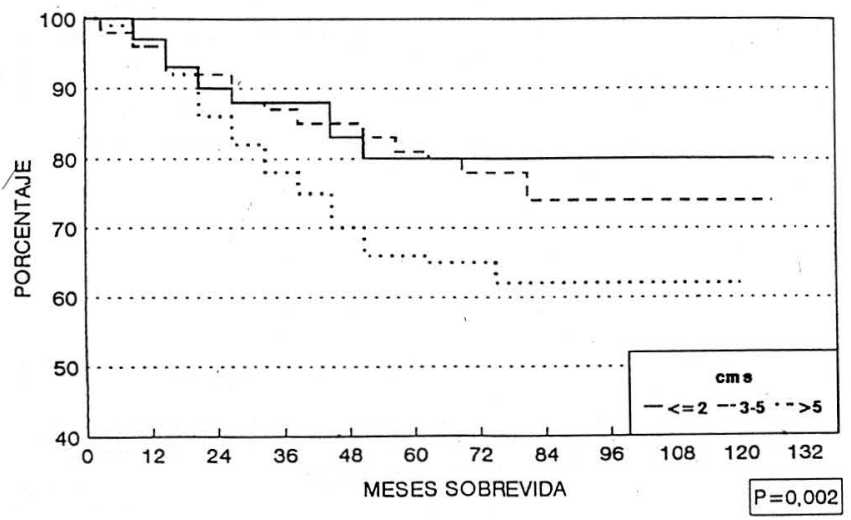

Figura 10

SOBREVIDA SEGUN EDAD DE LA MENOPAUSIA

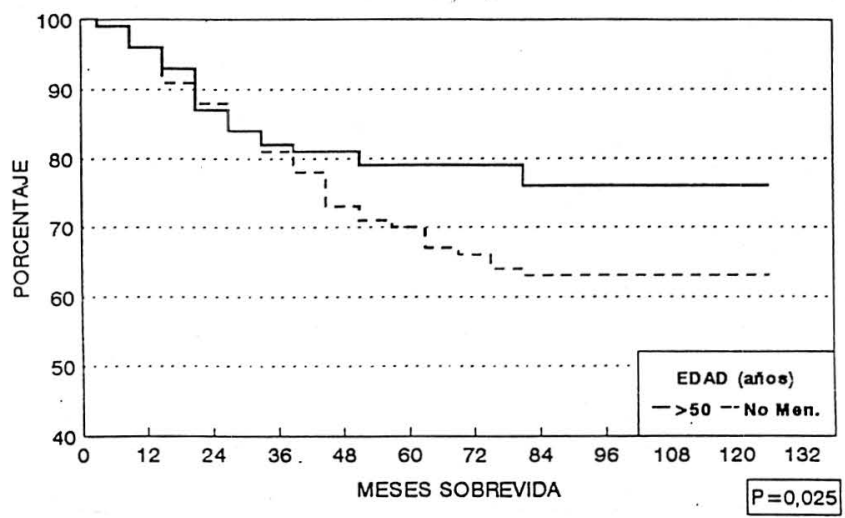

Tabla 8

PRONOSTICO SEGUN CARACTERISTICAS DE LAS PACIENTES SOBREVIDA

\begin{tabular}{|c|c|c|c|c|c|}
\hline \multirow{2}{*}{\multicolumn{2}{|c|}{ CARACTERISTICA }} & \multirow{3}{*}{$\frac{\mathrm{N}}{215}$} & \multicolumn{2}{|c|}{ VIVAS A 5 AÑOS } & \multirow{2}{*}{ Valor $P$} \\
\hline & & & $\%$ & $\mathrm{~N}$ & \\
\hline \multirow{2}{*}{ EDAD } & $\leq 45$ años & & 69,5 & 149 & \multirow[b]{2}{*}{0,055} \\
\hline & $>45$ años & 266 & 77,2 & 205 & \\
\hline \multirow{2}{*}{ EDAD MENARQUIA } & $\leq 12$ años & 135 & 68,0 & 92 & \multirow[b]{2}{*}{0.098} \\
\hline & $>12$ aĩos & 336 & 75,6 & 254 & \\
\hline \multirow{2}{*}{$\begin{array}{l}\text { ANTECEDENTES } \\
\text { FAMILIARES }\end{array}$} & Positivos & 53 & 81,4 & 43 & \multirow[b]{2}{*}{0.100} \\
\hline & Negativos & 373 & 70,4 & 262 & \\
\hline \multirow{4}{*}{$\begin{array}{l}\text { TIPO HISTOPATOLO- } \\
\text { GICO }\end{array}$} & Papilar* & 27 & 100,0 & 27 & \multirow[b]{4}{*}{0,109} \\
\hline & Lobulillar* & 33 & Insuf & & \\
\hline & Ductal & 345 & 70,3 & 243 & \\
\hline & Otro & 22 & 87,5 & 19 & \\
\hline \multirow{3}{*}{$\begin{array}{l}\text { NUMERO DE GESTA- } \\
\text { CIONES }\end{array}$} & Ninguria & 121 & 69,5 & 84 & \multirow[b]{3}{*}{0,288} \\
\hline & $1-3$ & 225 & 76.8 & 173 & \\
\hline & $>3$ & 118 & 71,9 & 85 & \\
\hline \multirow{2}{*}{$\begin{array}{l}\text { EDAD AL PRIMER } \\
\text { PARTO }\end{array}$} & $\leq 20$ años & 192 & 74,6 & 143 & \multirow[b]{2}{*}{0,564} \\
\hline & $>20$ años & 149 & 77.1 & 85 & \\
\hline \multirow{4}{*}{$\begin{array}{l}\text { LOCALIZACION CUA- } \\
\text { DRANTE UNICO }\end{array}$} & CSE & 205 & 77,3 & 159 & \multirow[b]{4}{*}{0,326} \\
\hline & CSI & 88 & 77.2 & 68 & \\
\hline & CII & 22 & 85,2 & 19 & \\
\hline & $\mathrm{ClE}$ & 39 & 67,5 & 26 & \\
\hline \multirow{4}{*}{$\begin{array}{l}\text { LOCALIZACION MUL- } \\
\text { TIPLES CUADRANTES }\end{array}$} & CSE - CSI & 50 & 63,9 & 32 & \multirow[b]{4}{*}{0.389} \\
\hline & CSI - CII & 4 & 33,3 & 1 & \\
\hline & $\mathrm{CII}-\mathrm{CIE}$ & 12 & 70,0 & 8 & \\
\hline & CSE - CIE & 10 & 54,0 & 5 & \\
\hline \multirow{2}{*}{ BILATERALIDAD } & Unilateral & 468 & 73,5 & 344 & \multirow[b]{2}{*}{0.470} \\
\hline & Bilateral & 13 & 68.2 & 9 & \\
\hline \multirow{2}{*}{ TIPO DE CIRUGIA } & MRM & 350 & 67,8 & 272 & \multirow[b]{2}{*}{0,565} \\
\hline & Cuadrantect. & 15 & 80,0 & 12 & \\
\hline \multirow{2}{*}{$\begin{array}{l}\text { RECEPTORES DE } \\
\text { PROGESTERONA }\end{array}$} & Positivos & 55 & 79,1 & 44 & \multirow[b]{2}{*}{0,624} \\
\hline & Negativos & 142 & 76,7 & 109 & \\
\hline \multirow{2}{*}{$\begin{array}{l}\text { RECEPTORES } \\
\text { ESTROGENICOS }\end{array}$} & Pusitives & 87 & 77,8 & 68 & \multirow[b]{2}{*}{0,874} \\
\hline & Negativess & 110 & 78.7 & 87 & \\
\hline
\end{tabular}

* No inctuidu en el análisis estallistie 
Tabla 9

SOBREVIDA A 10 AÑOS SEGUN CARACTERISTICAS DE LAS PACIENTES

\begin{tabular}{|c|c|c|c|c|c|c|c|c|}
\hline \multicolumn{2}{|c|}{ CARACTERISTICA } & $\begin{array}{c}6 \mathrm{~m} \\
\%\end{array}$ & $\begin{array}{c}12 \mathrm{~m} \\
\%\end{array}$ & $\begin{array}{c}24 \mathrm{~m} \\
\%\end{array}$ & $\begin{array}{c}36 \mathrm{~m} \\
\%\end{array}$ & $\begin{array}{c}60 \mathrm{~m} \\
\%\end{array}$ & $\begin{array}{c}96 m \\
\%\end{array}$ & $\begin{array}{c}120 \mathrm{~m} \\
\%\end{array}$ \\
\hline \multirow{6}{*}{ ESTADO } & I & 100,0 & 100,0 & 100,0 & 100,0 & 100,0 & 100,0 & 100,0 \\
\hline & IIA & 100,0 & 98,7 & 97,4 & 91,4 & 87,3 & 79,1 & 79,1 \\
\hline & IIB & 98,9 & 96,7 & 90,5 & 89.0 & 81,5 & 74,5 & 74,5 \\
\hline & IIIA & 100,0 & 99,1 & 92,8 & 87,8 & 80,3 & 72,0 & \\
\hline & IIIB & 98,2 & 94,3 & 79,5 & 68,4 & 50,8 & 50,8 & 50,8 \\
\hline & IV & 90,9 & 77,4 & 51,5 & 34,3 & 25.8 & 25,8 & \\
\hline \multirow{4}{*}{ ESTADO } & I & 100,0 & 100,0 & 100.0 & 100.0 & 100,0 & 100,0 & 100,0 \\
\hline & II & 90,4 & 97,6 & 93,7 & 90,1 & 84,2 & 77,0 & 77,0 \\
\hline & III & 99,1 & 96,7 & 86,1 & 78,1 & 65,4 & 61,4 & 61,4 \\
\hline & IV & 90,9 & 77,4 & 51,5 & 34,3 & 25,8 & 25,8 & \\
\hline \multirow{3}{*}{$\begin{array}{l}\text { GANGLIOS + } \\
\text { NIVEL } 1\end{array}$} & 0 & 100,0 & 100.0 & 97,8 & 95.3 & 92,6 & 87,8 & 87,8 \\
\hline & $1-3$ & 98,7 & 97,3 & 89,5 & 87,8 & 82,7 & 78,5 & 78,5 \\
\hline & $>3$ & 99,0 & 95,8 & 87,1 & 74,8 & 55,6 & 50,1 & 50,1 \\
\hline \multirow{3}{*}{$\begin{array}{l}\text { GANGLIOS + } \\
\text { NIVEL } 2\end{array}$} & 0 & 100,0 & 99,2 & 96,6 & 92,4 & 87,7 & 81,7 & 81.7 \\
\hline & $1-3$ & 97,1 & 97.1 & 90.7 & 83.2 & 73.9 & 61,6 & \\
\hline & $>3$ & 98.4 & 95.0 & 82,7 & 72,0 & 58.8 & 58,8 & \\
\hline \multirow{3}{*}{$\begin{array}{l}\text { GANGLIOS + } \\
\text { NIVEL } 3\end{array}$} & 0 & 99.3 & 99.3 & 92.5 & 89,6 & 86,4 & 82,7 & 82,7 \\
\hline & $1-3$ & 100,0 & 100,0 & 94,6 & 77,8 & 64,5 & 48,4 & \\
\hline & $>3$ & 100,0 & 92,0 & 79,8 & 79,8 & 57,1 & & \\
\hline \multirow{4}{*}{$\begin{array}{l}\text { TOTAL GAN- } \\
\text { GLIOS + }\end{array}$} & 0 & 100,0 & 100,0 & 97,8 & 94,4 & 91,6 & 86.8 & 86,8 \\
\hline & $1-3$ & 98,4 & 96.8 & 88.0 & 86,0 & 80.1 & 74,8 & 74,8 \\
\hline & $4-12$ & 100,0 & 100.0 & 93.9 & 79.1 & 69,2 & 62,3 & 62,3 \\
\hline & 213 & 98.0 & 91,8 & 81,2 & 76.5 & 53,8 & 53,8 & \\
\hline \multirow{4}{*}{$\begin{array}{l}\text { TIPO DE } \\
\text { RECAIDA }\end{array}$} & $L$ única & 100,0 & 100,0 & 90,9 & 85,5 & 78.9 & 67.7 & 67.7 \\
\hline & L nuủlt. & 100,0 & 100,0 & 66,7 & 33,3 & 33,3 & & \\
\hline & $M$ única & 96.2 & 86,9 & 68,5 & 55,3 & 31,6 & 11,5 & \\
\hline & $M$ mult. & 94,9 & 87,0 & 53,4 & 40,2 & 14,6 & & \\
\hline \multirow{3}{*}{$\begin{array}{l}\text { TAMAÑO DEL } \\
\text { TUMOR }\end{array}$} & $\leq 2 \mathrm{cms}$ & 100.0 & 96,9 & 90,0 & 88,1 & 79,6 & 79,6 & 79,6 \\
\hline & $3-5 \mathrm{cms}$ & 97,7 & 96.0 & 92,1 & 86,9 & 81.5 & 74,2 & 74,2 \\
\hline & $>5 \mathrm{cms}$ & 99,5 & 96,3 & 86,1 & 77,7 & 66,3 & 62,2 & 62,2 \\
\hline \multirow{3}{*}{$\begin{array}{l}\text { EDAD A LA } \\
\text { MENOPAUSIA }\end{array}$} & $\leq 50 \mathrm{a}$. & \multicolumn{7}{|c|}{ Información insuficiente para el cálculo de sobrevida } \\
\hline & $>50 \mathrm{a}$ & 98.4 & 96.1 & 87.4 & 82.3 & 78.9 & 75,5 & 75.5 \\
\hline & No & 98.8 & 95,6 & 87.6 & 80,8 & 69.8 & 62,6 & 62.6 \\
\hline \multirow{2}{*}{ EDAD } & $\leq 45$ a. & 99.1 & 96.6 & 87,4 & 79.9 & 69.5 & 60.7 & 60,7 \\
\hline & $>45$ a. & 98,4 & 95,6 & 88,6 & 83,7 & 77,2 & 75,1 & 75,1 \\
\hline \multirow{2}{*}{$\begin{array}{l}\text { EDAD A LA } \\
\text { MENARQUIA }\end{array}$} & $\leq 12$ a. & 97.0 & 94,6 & 83,5 & 75,7 & 68,0 & 61,3 & 61,3 \\
\hline & $>12$ a. & 99.4 & 96,5 & 89,7 & 84,1 & 75,6 & 70,3 & 70,3 \\
\hline \multirow{2}{*}{$\begin{array}{l}\text { ANTECEDENT. } \\
\text { FAMILIARES }\end{array}$} & Positiv. & 98.1 & 94.2 & 87,6 & 85,0 & 81,4 & 81,4 & \\
\hline & Negativ. & 98.9 & 96,0 & 86.5 & 80.3 & 70,4 & 65,0 & 65,0 \\
\hline \multirow{4}{*}{$\begin{array}{l}\text { TIPO HISTOPA- } \\
\text { TOLOGICO }\end{array}$} & Papilar & 100,0 & 100,0 & 100,0 & 100,0 & 100,0 & 100,0 & \\
\hline & Lobulil. & \multicolumn{7}{|c|}{ Información insuficiente para el cálculo de sobrevida } \\
\hline & Ductal & 98.8 & 96,0 & 86.5 & 79,8 & 70.3 & 64,0 & 64,0 \\
\hline & Ouro & 100.0 & 100.0 & 100.0 & 87.5 & 87.5 & 87.5 & \\
\hline & Ninguna & 98.3 & 96.6 & 88.0 & 82.4 & 69.5 & 67.0 & 67.0 \\
\hline GESTACIONES & $1-3$ & 99.1 & 96,7 & 89,0 & 81,8 & 76,8 & 70.6 & 70.6 \\
\hline & $>3$ & 98,2 & 93.6 & 85,8 & 80.3 & 71,9 & 59,6 & 59.6 \\
\hline EDAD AL PRI- & $\leq 20 \mathrm{a}$. & 98.4 & 95.5 & 90.5 & 82.1 & 74.6 & 63,0 & 63,0 \\
\hline MER PARTO & $>20 \mathrm{a}$ & 99.3 & 95.7 & 84.7 & 80.8 & 77.1 & 74.3 & 74.3 \\
\hline & CSE & 99.0 & 97.4 & 91,6 & 85,3 & 77,4 & 71,0 & 71.0 \\
\hline LOCALIZACION & $\mathrm{CSI}$ & 97.7 & 95.3 & 88.6 & 80.9 & 77.2 & 69.5 & 69.5 \\
\hline $\begin{array}{l}\text { CUADRANTE } \\
\text { UNICO }\end{array}$ & $\mathrm{CII}$ & 100,0 & 95,2 & 85,2 & 85,2 & 85.2 & 85.2 & \\
\hline & CIE & 100,0 & 88,8 & 80,2 & 77,2 & 67,5 & 67,5 & \\
\hline & CSE-SI & 98.0 & 98.0 & 91,3 & 83,2 & 63.9 & 55,9 & \\
\hline LOCALIZACION & CSI-II & 100.0 & $66: 7$ & 66.7 & 66.7 & 33,3 & 33,3 & \\
\hline $\begin{array}{l}\text { MULTIPLES } \\
\text { CUADRANTES }\end{array}$ & CII-IE & 100,0 & 100,0 & 70.0 & 70.0 & 70.0 & 70.0 & \\
\hline & CSE-IE & 100.0 & 90,0 & 67.5 & 67.5 & 54,0 & 54,0 & \\
\hline
\end{tabular}

\begin{tabular}{||l|l|l|l|l|l|l|l|l||}
\hline \multirow{3}{*}{ SECUENCIA } & $1^{\circ}$ prim. & 98,7 & 95,9 & 88,3 & 82,2 & 73,5 & 68,0 & 68,0 \\
\cline { 2 - 10 } & $2^{\circ}$ prim. & 100,0 & 100,0 & 81,8 & 68,2 & 68,2 & & \\
\hline \multirow{2}{*}{$\begin{array}{l}\text { TIPO DE } \\
\text { CIRUGIA }\end{array}$} & MRM & 99,4 & 97,9 & 92,1 & 87,2 & 77,8 & 72,6 & 72,6 \\
\cline { 2 - 10 } & Cuadr. & 100,0 & 100,0 & 93,3 & 80,0 & 80,0 & 80,0 & 80,0 \\
\hline $\begin{array}{l}\text { RECEPTORES DE } \\
\text { PROGESTERONA }\end{array}$ & Positiv. & 98,0 & 98,0 & 88,8 & 86,2 & 79,1 & 65,9 & \\
\cline { 2 - 10 } & Negativ. & 97,9 & 96,4 & 87,9 & 82,0 & 76,7 & 71,8 & 71,8 \\
\hline \multirow{2}{*}{$\begin{array}{l}\text { RECEPTORES } \\
\text { ESTROGENICOS }\end{array}$} & Positiv. & 97,6 & 96,4 & 86,6 & 82,0 & 77,8 & 68,2 & \\
\cline { 2 - 10 } & Negativ. & 98,1 & 97,1 & 88,3 & 83,8 & 78,7 & 74,8 & 74,8 \\
\hline \hline GLOBAL & & 98,7 & 96,0 & 88,1 & 81,9 & 73,4 & 67,9 & 67,9 \\
\hline
\end{tabular}

Tabla 10

PRONOSTICO SEGUN CARACTERISTICAS DE LAS PACIENTES TIEMPO LIBRE DE ENFERMEDAD

\begin{tabular}{|c|c|c|c|c|c|}
\hline \multirow{2}{*}{\multicolumn{2}{|c|}{ CARACTERISTICA }} & \multirow{3}{*}{$\begin{array}{l}\mathrm{N} \\
28\end{array}$} & \multicolumn{2}{|c|}{ LIBRES A 5 AÑOS } & \multirow{2}{*}{ Valor $\mathbf{P}$} \\
\hline & & & \multirow{2}{*}{$\begin{array}{l}\% \\
89,3\end{array}$} & \multirow{2}{*}{$\frac{N}{25}$} & \\
\hline \multirow{6}{*}{ ESTADO } & 1 & & & & \multirow[b]{6}{*}{$<0.0001$} \\
\hline & IIA & 87 & 86,0 & 75 & \\
\hline & $11 \mathrm{~B}$ & 93 & 67,5 & 63 & \\
\hline & IIIA & 113 & 59,9 & 68 & \\
\hline & IIIB & 113 & 38,0 & 43 & \\
\hline & IV & 34 & 0 & 0 & \\
\hline \multirow{4}{*}{ ESTADO } & 1 & 28 & 89,3 & 25 & \multirow[b]{4}{*}{$<0,0001$} \\
\hline & II & 180 & 75,8 & 136 & \\
\hline & III & 226 & 48,8 & 110 & \\
\hline & IV & 34 & 0 & 0 & \\
\hline \multirow{3}{*}{$\begin{array}{l}\text { GANGLIOS POSITIVOS } \\
\text { NIVEL } 1\end{array}$} & 0 & 154 & 84,9 & 131 & \multirow[b]{3}{*}{$<0,0001$} \\
\hline & $1-3$ & 77 & 59.6 & 46 & \\
\hline & $>3$ & 98 & 44,0 & 43 & \\
\hline \multirow{3}{*}{$\begin{array}{l}\text { GANGLIOS POSITIVOS } \\
\text { NIVEL } 2\end{array}$} & 0 & 128 & 76,7 & 98 & \multirow[b]{3}{*}{$<0.0001$} \\
\hline & $1-3$ & 35 & 67.6 & 24 & \\
\hline & $>3$ & 62 & 39,8 & 25 & \\
\hline \multirow{3}{*}{$\begin{array}{l}\text { GANGLIOS POSITIVOS } \\
\text { NIVEL } 3\end{array}$} & 0 & 137 & 79.8 & 109 & \multirow[b]{3}{*}{$<0,0001$} \\
\hline & $1-3$ & 43 & 49.1 & 21 & \\
\hline & $>3$ & 25 & 32.2 & 8 & \\
\hline \multirow{4}{*}{$\begin{array}{l}\text { TOTAL GANGLIOS } \\
\text { POSITIVOS }\end{array}$} & 0 & 153 & 84,8 & 130 & \multirow{4}{*}{$<0.0001$} \\
\hline & $1-3$ & 64 & 60,7 & 39 & \\
\hline & $4-12$ & 77 & 56,3 & 43 & \\
\hline & $\geq 13$ & 49 & 35,4 & 17 & \\
\hline \multirow{3}{*}{ TAMAÑO DEL TUMOR } & $\leq 2 \mathrm{cms}$ & 70 & 74,9 & 52 & \multirow{3}{*}{$<0,0001$} \\
\hline & $3-5 \mathrm{cms}$ & 182 & 67,8 & 123 & \\
\hline & $>5 \mathrm{cms}$ & 199 & 49,0 & 98 & \\
\hline \multirow{4}{*}{ TIPO DE RECAIDA } & Local única & 25 & 22,9 & 6 & \multirow[b]{4}{*}{0,006} \\
\hline & Local múltiple & 3 & 0 & 0 & \\
\hline & Metást. única & 80 & 5,2 & 4 & \\
\hline & Metást. múltip. & 40 & 3.6 & 1 & \\
\hline \multirow{4}{*}{ TIPO HISTOPATOLOGICO } & Papilar & 27 & 65,8 & 18 & \multirow[b]{4}{*}{0,012} \\
\hline & Lobulillar & 33 & 78,7 & 26 & \\
\hline & Ductal & 345 & 57,0 & 197 & \\
\hline & Otro & 22 & 79,9 & 18 & \\
\hline
\end{tabular}


Figura 11

TIEMPO LIBRE DE ENFERMEDAD GLOBAL

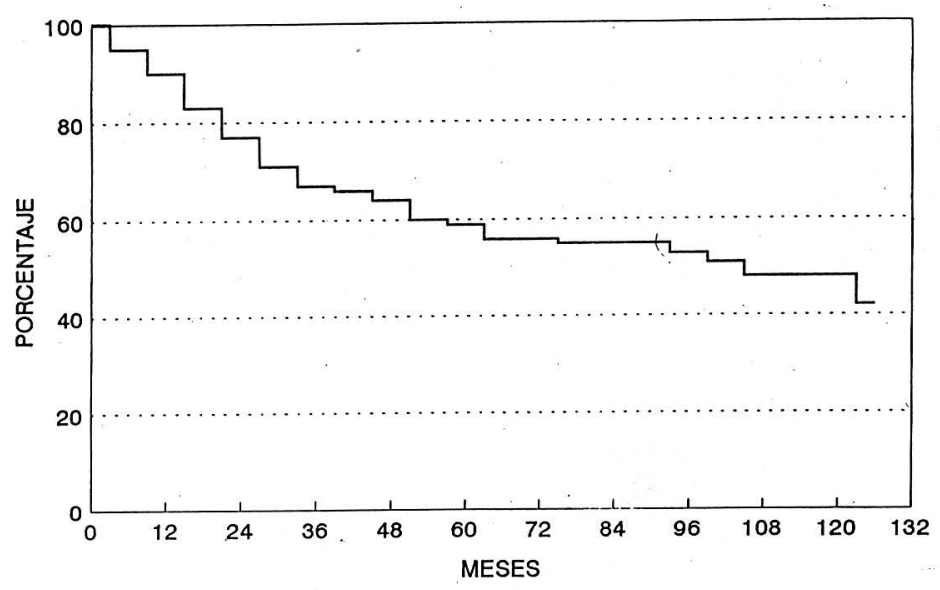

Figura 12

TIEMPO LIBRE DE ENFERMEDAD SEGUN ESTADO

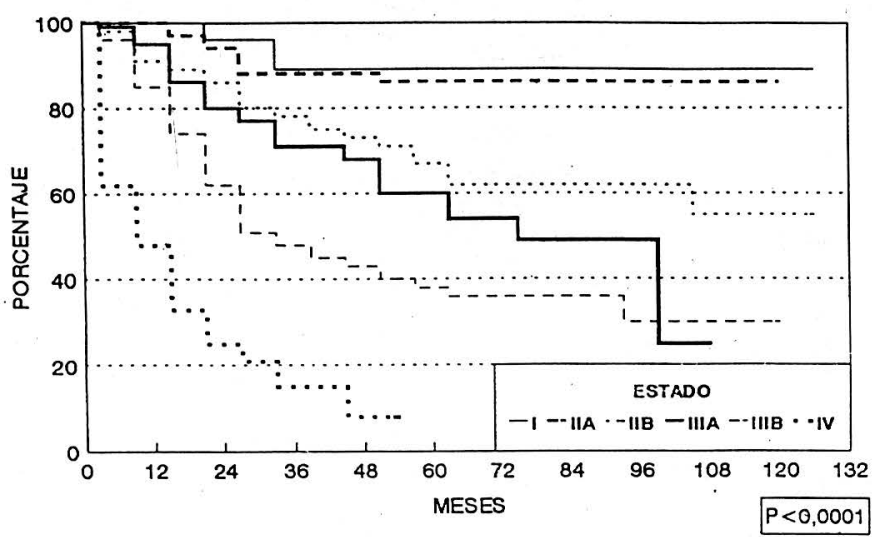

Figura 13

TIEMPO LIBRE DE ENFERMEDAD SEGUN ESTADO

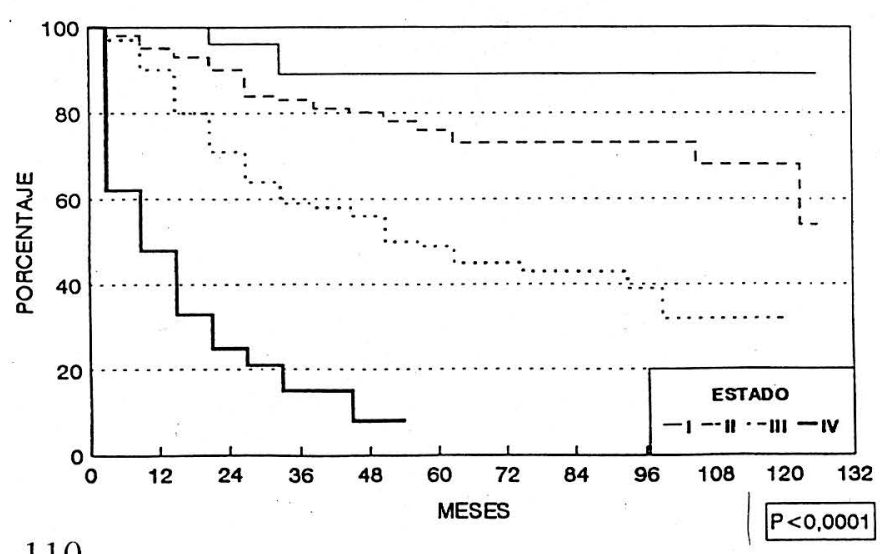

El diagnóstico histopatológico de carcinoma ductal infiltrante mostró un mayor compromiso a los cinco años, $43 \%$ en comparación con los otros tipos histopatológicos $\mathrm{P}<0,012$.

Otras variables analizadas para el tiempo libre de enfermedad se encuentran en la tabla 11. Estas últimas no presentaron diferencias significativas con respecto al atributo analizado.

El tiempo libre enfermedad global se presenta en la figura 2 , se aprecia que la recaída es mayor en los dos primeros años.

\section{Discusión}

La extensión anatómica del cáncer evaluado de acuerdo con la clasificación por estados se considera como un parámetro pronóstico significativo en el cáncer de mama.

La Comisión Conjunta del Cáncer de los Estados Unidos (AJCC) y la Unión Internacional contra el Cáncer (UICC) acordaron establecer un nuevo sistema TNM para la mama (7).

Los estados son enumerados desde el 0 hasta el IV, del mejor al peor pronóstico con base en los índices de sobrevida.

El cambio conlleva, sin embargo, que la evaluación de estudios previos a su aceptación presentan dificultades para homologarlos con los que puedan surgir posteriormente (10).

Además de esta dificultad una clasificación por estados apropiada requiere fundamentalmente de una evaluación patológica más que de una evaluación clínica de los ganglios linfáticos, pues ésta última es inexacta y se ha aceptado que existe una diferencia de aproximadamente un $33,8 \%$ entre las dos (10).

Con estas salvedades se pudo determinar en el grupo de pacientes estudiadas una relación significativa estadísticamente $(\mathrm{P}<0,0001)$ entre el estado clínico y el pronóstico de la enfermedad al determinar la sobrevida y el tiempo libre de enfermedad, lo cual recalca la necesidad de realizar continuos esfuerzos para establecer una detección temprana del cáncer de mama con el fin de obtener un mejor resultado terapéutico $(3,14)$.

El número de ganglios axilares comprometidos así como el nivel de la metástasis ganglionares resultaron estadísticamente significativas $(\mathrm{P}<0,0001)$ en el análisis de sobrevida y tiempo libre de enfermedad. La sobrevida a cinco (5) años se reduce del $91,6 \%$ cuando no existe compromiso axilar al $53,8 \%$ cuando hay compromiso tumoral de 13 ganglios o más (Tabla 7), siendo éste un resultado similar al de otros estudios.

En un estudio de 20.547 pacientes con cáncer de mama realizado por el Colegio Americano de Cirujanos, la sobrevida disminuyó del $71,6 \%$ al 29\% cuando existía compromiso tumoral de 21 ganglios o más $(2,13)$.

El parámetro pronóstico de compromiso ganglionar axilar tiene que ver con la interacción existente entre huésped y tumor que permite su diseminación metastásica de acuerdo con la hipótesis alternativa de Fisher (8).

El tamaño tumoral mostró significancia estadística $P=0.002$ señalando que presumiblemente el mayor ta- 
Tabla 11

PRONOSTICO SEGUN CARACTERISTICAS DE LAS PACIENTES - TIEMPO LIBRE DE ENFERMEDAD

\begin{tabular}{|c|c|c|c|c|c|}
\hline \multirow{2}{*}{\multicolumn{2}{|c|}{ CARACTERISTICA }} & \multirow{3}{*}{$\begin{array}{c}\mathrm{N} \\
53\end{array}$} & \multicolumn{2}{|c|}{ LIBRES A 5 AÑOS } & \multirow{2}{*}{ Vaḷor $\mathbf{P}$} \\
\hline & & & \multirow{2}{*}{$\begin{array}{l}\% \\
67,1\end{array}$} & \multirow{2}{*}{ N } & \\
\hline ANTECEDENTES & Positivos & & & & \multirow[b]{2}{*}{0,064} \\
\hline & Negativos & 373 & 54,4 & 203 & \\
\hline \multirow{2}{*}{ EDAD } & $\leq 45$ años & 215 & 55,1 & 119 & \multirow[b]{2}{*}{0,117} \\
\hline & $>45$ años & 266 & 62,5 & 166 & \\
\hline \multirow{3}{*}{ EDAD MENOPAUSIA } & $\leq 50$ años & 13 & 70,7 & s & \multirow[b]{3}{*}{0.118} \\
\hline & $>50$ años & 192 & 63,9 & 123 & \\
\hline & No menopáus. & 265 & 54,9 & 146 & \\
\hline \multirow{2}{*}{$\begin{array}{l}\text { EDAD AL PRIMER } \\
\text { PARTO }\end{array}$} & $\leq 20$ años & 192 & 54,3 & 104 & \multirow[b]{2}{*}{0,160} \\
\hline & $>20$ años & 149 & 61,6 & 92 & \\
\hline \multirow{3}{*}{$\begin{array}{l}\text { NUMERO DE } \\
\text { GESTACIONES }\end{array}$} & Ninguna & 121 & 61,3 & 74 & \multirow[b]{3}{*}{0,319} \\
\hline & $1-3$ & 225 & 59,9 & 135 & \\
\hline & $>3$ & 118 & 52,5 & 62 & \\
\hline \multirow{2}{*}{$\begin{array}{l}\text { EDAD } \\
\text { MENARQUIA }\end{array}$} & $\leq 12$ años & 135 & 56,5 & 76 & \multirow{2}{*}{0,482} \\
\hline & $>12$ años & 336 & 59,8 & 201 & \\
\hline \multirow{2}{*}{$\begin{array}{l}\text { RECEPTORES DE } \\
\text { PROGESTERONA }\end{array}$} & Positivos & 55 & 47,8 & 26 & \multirow[b]{2}{*}{0,110} \\
\hline & Negativos & 142 & 59,8 & 85 & \\
\hline \multirow{2}{*}{$\begin{array}{l}\text { RECEPTORES } \\
\text { ESTROGENICOS }\end{array}$} & Positivos & 87 & 53.4 & 46 & \multirow[b]{2}{*}{0,382} \\
\hline & Negativos & 110 & 59,3 & 85 & \\
\hline \multirow{2}{*}{ TIPO DE CIRUGIA } & MRM & 350 & 68,2 & 239 & \multirow[b]{2}{*}{0,339} \\
\hline & Cuadrantec. & 15 & 62,5 & 9 & \\
\hline \multirow{4}{*}{$\begin{array}{l}\text { LOCALIZACION } \\
\text { CUADRANTE UNICO }\end{array}$} & CSE & 205 & 61,7 & 126 & \multirow[b]{4}{*}{0,357} \\
\hline & CSI & 88 & 66,7 & 59 & \\
\hline & $\mathrm{CII}$ & $22:$ & 67,2 & 15 & \\
\hline & $\mathrm{CIE}$ & 39 & 51,0 & 20 & \\
\hline \multirow{2}{*}{ BILATERALIDAD } & Unilateral & 468 & 56,1 & 262 & \multirow{2}{*}{0,691} \\
\hline & Bilateral & 1.3 & 59.1 & 8 & \\
\hline \multirow{4}{*}{$\begin{array}{l}\text { LOCALIZACION MUL- } \\
\text { TIPLES CUADRANTES }\end{array}$} & CSE - CSI & 50 & 59,3 & 30 & \multirow[b]{4}{*}{0,930} \\
\hline & CSI - CII & 4 & 37,5 & 2 & \\
\hline & CII - CIE & 12 & 58,3 & 7 & \\
\hline & CSE - CIE & 10 & 53,3 & 5 & \\
\hline
\end{tabular}

Figura 14

TIEMPO LIBRE DE ENFERMEDAD SEGUN NUMERO DE GANGLIOS POSITIVOS NIVEL 1

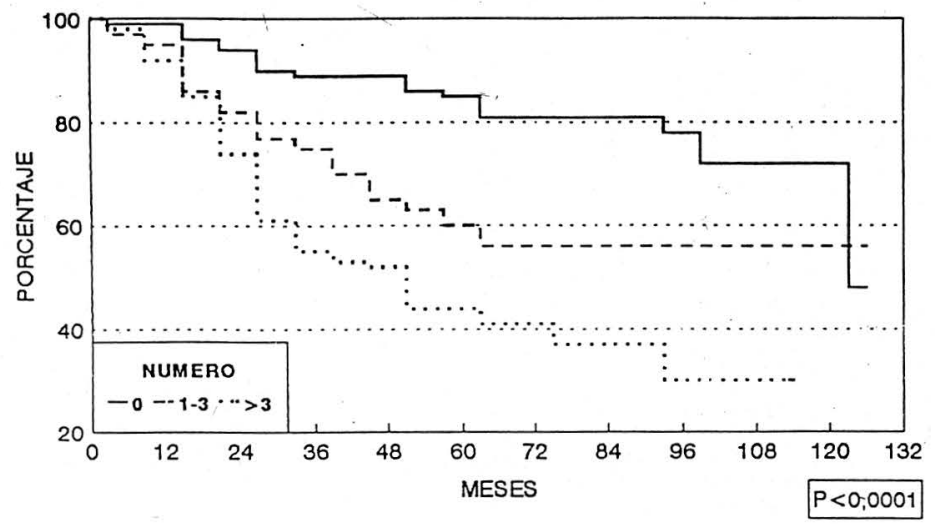

Figura 15

TIEMPO LIBRE DE ENFERMEDAD SEGUN NUMERO DE GANGLIOS POSITIVOS NIVEL 2

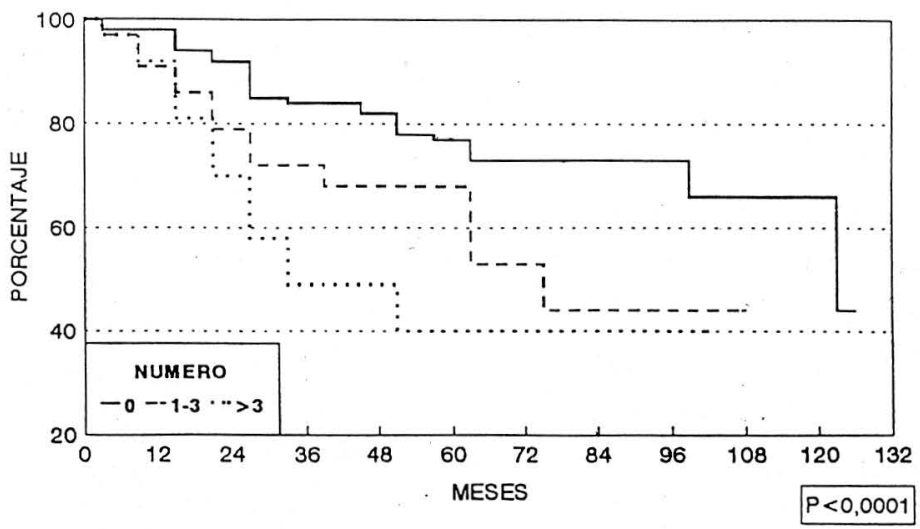

Figura 16

TIEMPO LIBRE DE ENFERMEDAD SEGUN NUMERO DE GANGLIOS POSITIVOS NIVEL 3

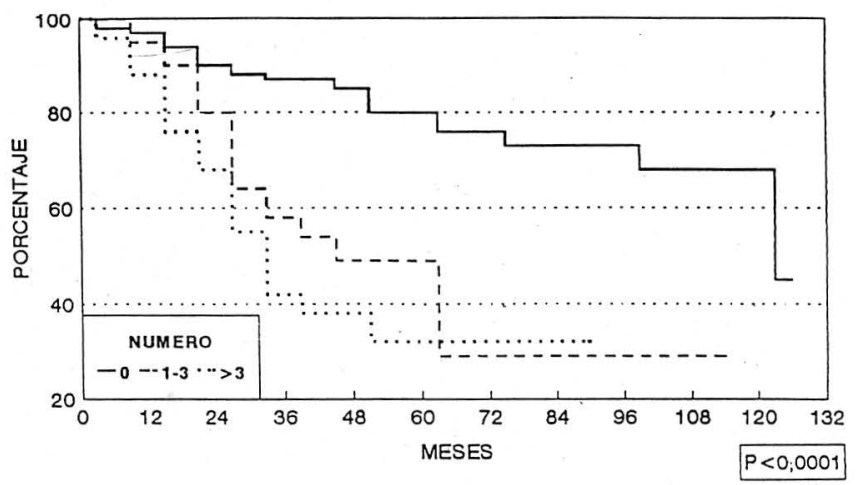

Figura 17

TIEMPO LIBRE DE ENFERMEDAD SEGUN TOTAL DE GANGLIOS POSITIVOS

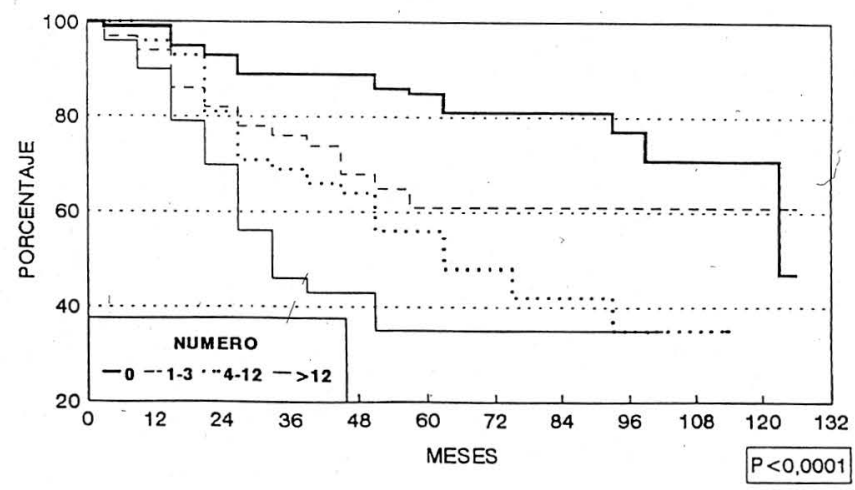


Figura 18

TIEMPO LIBRE DE ENFERMEDAD SEGUN TAMAÑO DEL TUMOR

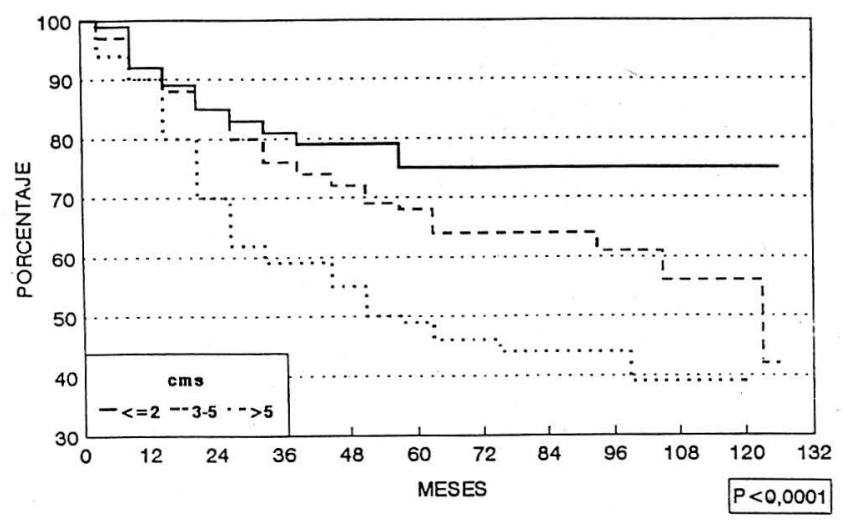

Figura 19

TIEMPO LIBRE DE ENFERMEDAD SEGUN TIPO DE RECAIDA

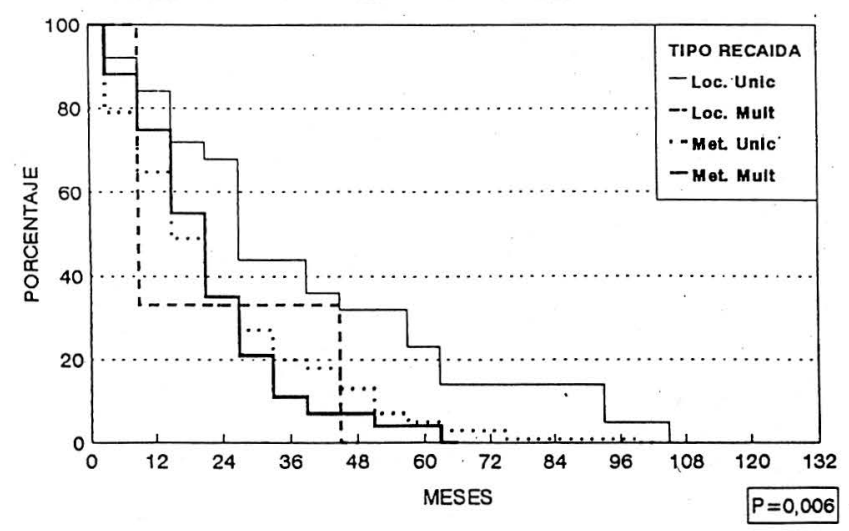

Figura 20

TIEMPO LIBRE DE ENFERMEDAD SEGUN TIPO HISTOPATOLOGICO

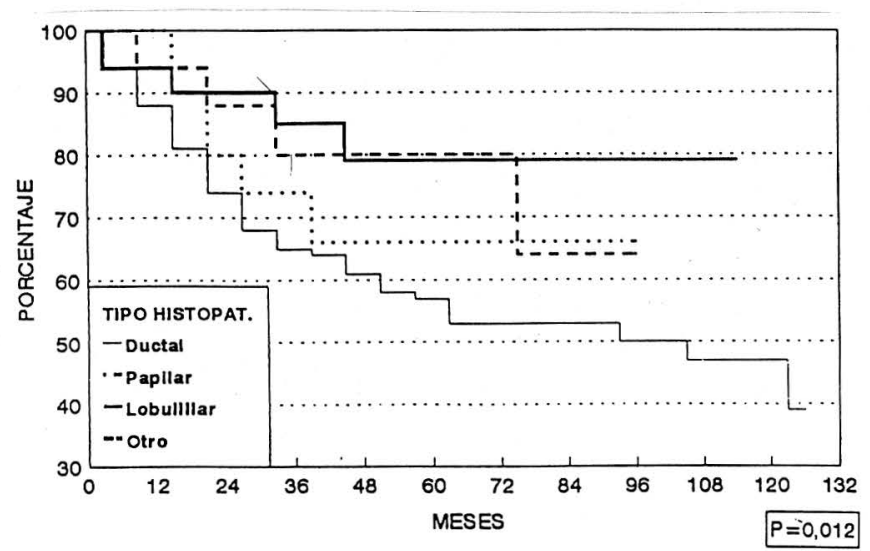

maño está relacionado con una tasa y duración de crecimientos mayores. Además, se ha encontrado una relación directa entre el tamaño tumoral y la posibilidad de compromiso metastásico en ganglios axilares, así como la presencia de multicentricidad. El carcinoma invasivo menor de $5 \mathrm{~mm}$. de diámetro está asociado con metástasis axilares en el $20 \%$ de las pacientes, este porcentaje se aumenta en el tumor de hasta $1 \mathrm{~cm}$. de diámetro al $27 \%$ y a casi el $80 \%$ cuando el tumor alcanza $10 \mathrm{cms}$. o más (1).

En el presente estudio esta asociación fue significativa estadísticamente con un valor de $\mathrm{P}<0,0001$, sin embargo, su impacto en el pronóstico no se fundamenta exclusivamente en el compromiso ganglionar como se ha visto en las pacientes con ganglios negativos. En un estudio del Colegio Americano de Cirujanos, se reporta un descenso del $82,2 \%$ al $56,8 \%$ cuando los tumores aumentaron de $0,5 \mathrm{~cm}$ ó menos a 5 ó más cms. de diámetro, sugiriendo vías diferentes a la linfática para su diseminación, como la vía sanguínea o a través del compartimento intersticial (13).

Cuando se evalúa el tamaño tumoral y es menor o hasta de $3 \mathrm{cms}$. es recomendable la medición exclusiva del compromiso invasivo, ya que muchos de estos tumores se acompañan de un componente intraductal (16).

El tipo histológico tuvo significancia estadística al evaluar la probabilidad de recurrencia constituyéndose el ductal infiltrante en un cáncer con mayor predisposición a recaer, ese comportamiento está asociado significativamente $\mathrm{P}<0,0001$ con un mayor compromiso de ganglios axilares. Este parámetro pronóstico se ha encontrado con igual importancia en otros reportes (6).

La búsqueda de factores pronósticos ha despertado un gran interés en determinar el factor ideal que permita una predicción más precisa y lleve a tres usos fundamentales;

a) Seleccionar un tratamiento adecuado para un determinado paciente.

b) Permitir comparaciones de tratamientos entre grupos de pacientes con riesgos semejantes de recurrencia o muerte.

c) Mejorar nuestro conocimiento del cáncer de mama con el desarrollo de nuevas estrategias investigativas.

\section{Conclusiones}

La revisión de pacientes con diagnóstico de cáncer de mama en la Clínica San Pedro Claver de Santafé de Bogotá (ISS) permitió establecer que los factores pronósticos con significancia estadística en el análisis de sobrevida y tiempo libre de enfermedad son los mismos determinados por otros estudios en sobrevida, estado, ganglios positivos y compromiso tumoral de acuerdo con la distribución por niveles, tipo de recaída, tamaño tumoral y edad de menopausia; y para tiempo libre de enfermedad, estado, ganglios positivos y su compromiso por niveles, tamaño del tumor, tipo de recaída y tipo histopatológico. Estos factores tienen valor en la decisión terapéutica e investigativa del carcinoma mamario. 


\section{BIBLIOGRAFIA}

1. Carter CL., Allen C., Henson DC. Relation of tumor size, lymph node status and survival in 24740 breast cancer cases. Cancer 1989; 63: 181-187.

2. Donegan WL. Pronostic factors, stage and receptor in Breast Cancer. Cancer 1992; 70: 1755-1764

3. Feig SA. Mammographic Screening of women aged 40 to 49 years. Obstetrics and Gynecology Clinics of North America. 1994; 21(4): 587-606.

4. Giuliano AE. Breast disease en Berek JS, Hacker NF. Practical Gynecologic Oncology, Editors, Baltimore: Williams \& Wilkins. 1994; 481-518.

5. González MA., Corso J., Sáenz MC., Posso HJ., Martínez G. Carcinoma invasivo de cervix. Instituto Nacional de Cancerología. 1985 1987. Revista Colombiana de Obstetricia y Ginecología. 1994; 45: $135-140$.

6. Harris JR., Morrow M., Bonadonna G. Cancer of the Breast. En de VTgr., Helmann S, Rosenberg SA. Cancer Principles and practice of Oncology, Philadelphia: JB Lippincott 1993; 1264-1332.

7. Hutter RVP. At last-worldwide agreement on the staging of Cancer. Arch Surg 1987; 122: 1235.

8. Kardinal CG. Chemotherapy of Breast Cancer. En Michael C. Perry the chemotherapy source book Baltimore: Williams \& Wilkins, 1992; 949-988.
9. Leis HP. JR. Epidemiología en el cáncer de mama. En Strombeck JO Rosato FE Cirugía de la mama. Barcelona Salvat Editores. 1990; 102 111.

10. Leis HP. GR. Parámetros pronósticos para el cáncer de mama. En Bland KI, Copelamnd IIIE. M. La mama manejo multidisciplinario de las enfermedades benignas y malignas. Buenos Aires, Editorial Médica Panamericana, 1993; 427-451.

11. Marchant PJ. Contemporary Management of breast cancer obstetrics and Gynecology clinics of North America 1994; 21(4): 555-560.

12. Miller WR., Ellis IO., Sainsbury JRC., Dixon JM. Pronostic factors British Medical Journal. 1994; 309 (6968): 1573-1576.

13. NemotoT., Vana J., Bedwan RN., Baker HW., McGregorFIT., Murphy GP. Management and Survival of Female Breast Cancer: results of a national survey by the American College of Surgeons. Cancer 1980; 45: 2917-2924.

14. Petrek JA. Breast Cancer. Clinical Obstetrics and Gynecology 1994; 37(4): 955-966.

15. Perry F. Carcinoma in situ de Seno. Revista Colombiana de Obstetricia y Ginecología 1995; 46(1): 11-16.

16. Seidman JD., Schnaper LA., Aisner SC. Relationship of the Size of the invasive component of the primary Breast Carcinøma to Axillary Lynph No de metástasis. Cancer 1995; 75: 65-71. 